\title{
PHOTOREFLECTANCE MEASUREMENTS OF InGaAs/GaAs QUANTUM WELLS
}

\author{
T. Tomaszewicz, K.P. Korona, R. BożeK and J.M. Baranowski
}

Institute of Exper. Physics, Warsaw University, Hoża 69, 00-681 Warszawa, Poland

Samples with InGaAs/GaAs quantum wells were grown by metallo-organic chemical vapour deposition in order to detect and analyze GaSb islands deposited on the surface. Results of photoreflectance measurements of quantum wells are reported. The correspondence between broadening of quantum well transition lines and GaSb structures has been observed.

PACS numbers: 68.55.Bd, 73.20.Dx, 78.66.Fd

\section{Introduction}

Recently quantum dots and low dimensional structures have been of great interest due to interesting physical phenomena and possibility for useful applications. Quantum dots while observing by microscope are difficult to see in photoluminescence and photoreflectance measurements. In order to help to analyze small $\mathrm{GaSb}$ structures on the surface a set of samples with quantum well under the GaSb dots was prepared.

\section{Experimental}

Samples were prepared by metallo-organic chemical vapour deposition (MOCVD) on [100] GaAs substrate. For all samples $750 \mathrm{~nm} \mathrm{GaAs}$ buffer was grown to improve a surface quality. Four samples were investigated:

1. $\mathrm{Ga}_{0.82} \mathrm{In}_{0.18}$ As quantum well (QW) of $50 \AA$ covered by $200 \AA$ GaAs barrier;

2. $\mathrm{Ga}_{0.82} \mathrm{In}_{0.18} \mathrm{As} \mathrm{QW}$ of $50 \AA$ covered by $120 \AA \mathrm{GaAs}$ barrier followed by $18 \AA \mathrm{GaSb}$;

3. $\mathrm{Ga}_{0.82} \operatorname{In}_{0.18} \mathrm{As} \mathrm{QW}$ of $50 \AA$ covered by $120 \AA \mathrm{GaAs}$ barrier followed by $36 \AA \mathrm{GaSb}$;

4. $\mathrm{Ga}_{0.82} \operatorname{In}_{0.18} \AA \mathrm{s} \mathrm{QW}$ of $50 \AA$ covered by $200 \AA \mathrm{GaAs}$ barrier followed by $36 \AA \mathrm{GaSb}$.

The samples were intentionally undoped, however the electron concentration in $\mathrm{GaAs}$ was about $2 \times 10^{15} \mathrm{~cm}^{-1}$ and the hole concentration in $\mathrm{GaSb}$ was about $2 \times 10^{17} \mathrm{~cm}^{-1}$ as deduced from the growth parameters.

Because of the lattice mismatch between $\mathrm{GaAs}$ and $\mathrm{GaSb}(7.8 \%)$ a thin strained layer of $\mathrm{GaSb}$ is unstable and after depositing a few monolayers $\mathrm{GaSb}$ relaxes and forms small anisotropic islands of typical dimensions $100 \times 200 \mathrm{~nm}$ in agreement with previous investigations [1]. The sample 1 with $50 \AA \mathrm{QW}$ but without $\mathrm{GaSb}$ islands was prepared for comparison. 
The influence of $\mathrm{GaSb}$ islands on energy states in QW was investigated using photo-voltaic spectroscopy (PVS) and photoreflectance measurements (PR) at room temperature. The PR measurements were performed using a GDM-1000 monochromator, a He-Ne laser and a Si detector.

\section{Experimental results}

The PVS results for the sample 3 are presented in Fig. 1. Successive curves correspond to various depths of etching of the layer. The curve for $d=0$ corresponds to an unetched layer, and the curve for $d=2 \mathrm{~nm}$ corresponds to the etching of the GaSb islands. The results of PVS show three maxima at 1.30, 1.32, $1.36 \mathrm{eV}$, corresponding to three transitions in QW.

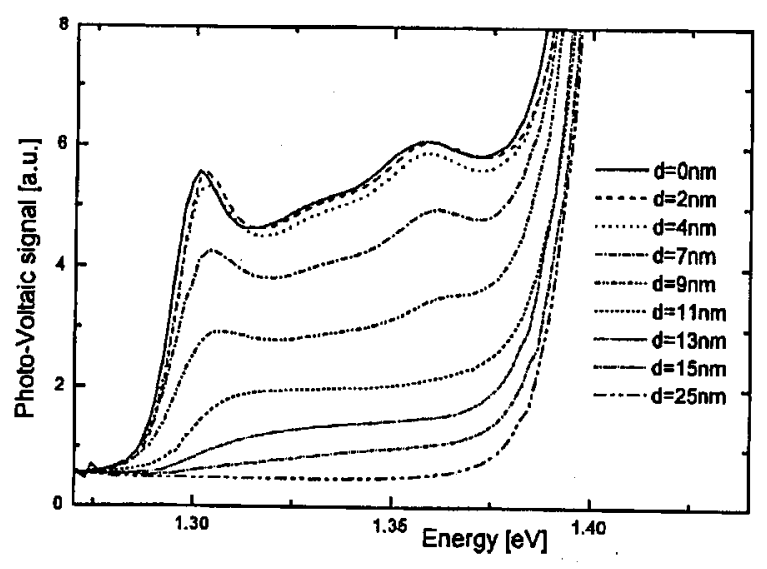

Fig. 1. Photo-voltaic spectroscopy results of the sample 3 with $120 \AA$ barrier and $36 \AA$ $\mathrm{GaSb}$ islands. Successive curves correspond to various depths of etching.

Energy positions of the first transition during etching for the sample 1 without $\mathrm{GaSb}$ and the sample 3 with GaSb islands are plotted in Fig. 2. It can be seen that removing $\mathrm{GaSb}$ islands produces a shift in a position of the first QW level by about $2 \mathrm{meV}$. This effect does not exist on the sample 1 without GaSb. Moreover the exponential dependence of energy versus barrier thickness has been observed. It is due to the exponential tail of wave functions of states in a finite quantum well: $\psi(x)=a \exp (-\kappa x)$, where $\kappa=\left(2 \Delta E_{m}\right)^{1 / 2} / 2 \pi h$.

The photoreflectance results in the energy range $1.27-1.33 \mathrm{eV}$ corresponding to the first QW state are shown for three samples in Fig. 3. Experimental results indicated as points and curves were calculated according to the following equation [2]:

$$
\mathrm{d} R / R=\operatorname{Re}\left[C \mathrm{e}^{\mathrm{i} \phi}\left(E-E_{0}+\mathrm{i} \Gamma\right)^{-n}\right]
$$

where $E_{0}$ is transition energy, $\Gamma-$ broadening parameter, $C$ and $\phi-$ amplitude and phase. The parameter $n=3$ for two-dimensional structures. An estimation of electric field $\varepsilon$ in the middle of $\mathrm{QW}$ was performed in a simple electrostatic model, 


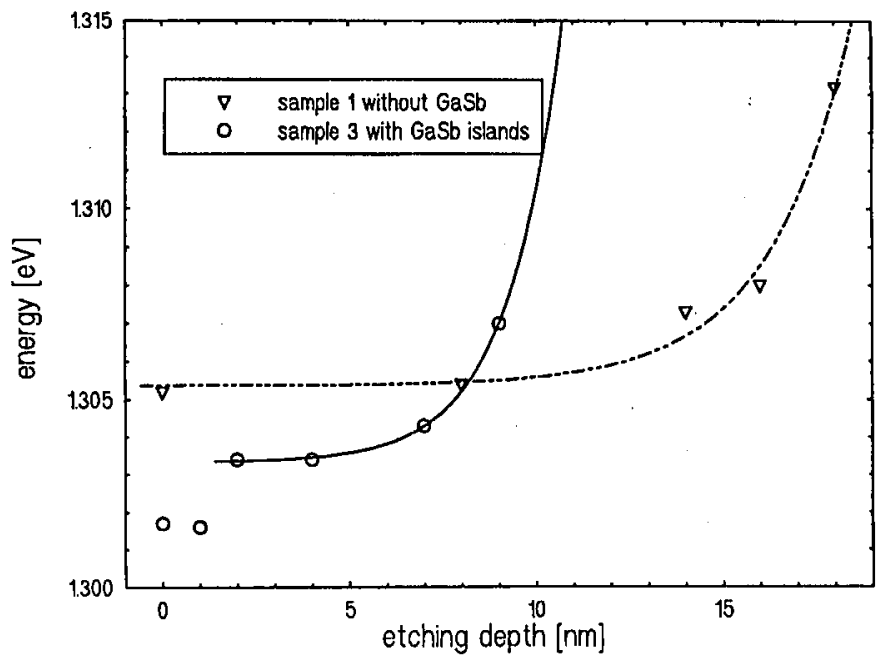

Fig. 2. Changes of the first transition energy in $Q W$ during etching. Results for the sample without $\mathrm{GaSb}$ and for the sample 3 (with $36 \AA \mathrm{GaSb}$ ). Solid lines are exponential functions plotted as guides for eyes.

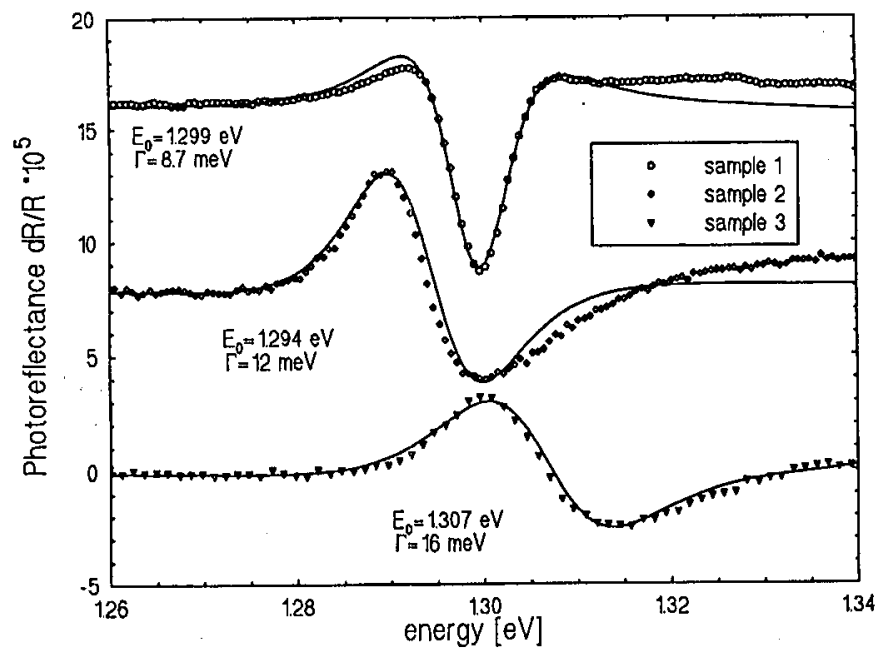

Fig. 3. Photoreflectance spectra of three investigated samples. Solid lines are fitted following Eq. (1).

taking into account that electrons from GaAs move to GaSb islands forming a depleted layer. An electric charge in the depleted layer can generate the electric field up to $10 \mathrm{kV} / \mathrm{cm}$. 
The fit of Eq. (1) to the experimental points and electric field $\varepsilon$ calculations give the following results:

Sample 1 (barrier: $200 \AA$; GaSb: $0 \AA$ ): $E_{0}=1.299 \mathrm{eV}, \Gamma=8.7 \mathrm{meV}, \varepsilon=0$;

Sample 2(barrier: $120 \AA$; GaSb: $18 \AA$ ): $E_{0}=1.294 \mathrm{eV}, \Gamma=12 \mathrm{meV}, \varepsilon=4.8 \mathrm{kV} / \mathrm{cm}$;

Sample 3 (barrier: $120 \AA$; GaSb: $36 \AA$ ): $E_{0}=1.307 \mathrm{eV}, \Gamma=16 \mathrm{meV}, \varepsilon=10.5 \mathrm{kV} / \mathrm{cm}$; Sample 4 (barrier: $200 \AA$; GaSb: $36 \AA$ ): $E_{0}=1.296 \mathrm{eV}, \Gamma=12 \mathrm{meV}, \varepsilon=10.2 \mathrm{kV} / \mathrm{cm}$.

For the samples with GaSb small transition energy shifts (from -5 to $+8 \mathrm{meV}$ ) in respect of $E_{0}$ for the sample 1 are observed, which can be due to uncertainty of QW width. On the other hand an increase in broadening parameter is clearly observed. A sample with $120 \AA \mathrm{GaAs}$ barrier has a larger broadening parameter than a sample with $200 \AA$ GaAs barrier. An increase in GaSb thickness also leads to broadening of the line. Moreover, it is visible that the line width increases with an increase in expected electric field.

\section{Discussion and conclusions}

Our results clearly indicate that the presence of $\mathrm{GaSb}$ islands influences on the energy of the fundamental state of QW. The change of energy position and particular of the broadening of this level is observed.

In principle there can be three reasons of these effects - strain, electric field and the effect of penetration of $\mathrm{QW}$ wave function into $\mathrm{GaSb}$ islands. Taking into account the $\mathrm{GaSb} / \mathrm{GaAs}$ lattice mismatch $(7.8 \%)$ and GaAs pressure band gap shift $\left(12.6 \times 10^{-6} \mathrm{eV} / \mathrm{bar}\right)[3]$ we can estimate that the band gap shift induced by the strain could be at most $1 \mathrm{meV}$. From the PVS spectra we can estimate the energy shift due to the finite barrier width. For the sample 3 with 120 barrier this shift is of the order of $0.03 \mathrm{meV}$. So, the best explanation of the broadening of PR lines can be the unhomogeneities of electric field in $\mathrm{Ga}_{0.82} \operatorname{In}_{0.18} \mathrm{As} Q W$ generated by GaSb islands.

\section{Acknowledgments}

This work was partially supported by European Community Grant CIPA CT 930162.

\section{References}

[1] R. Bożek, A. Babiński, J.M. Baranowski, R. Stępniewski, Z. Klusek, W. Olejniczak, K. Starowieyski, J. Wróbel, Acta Phys. Pol. A 88, 974 (1995).

[2] D.E. Aspnes, Surf. Sci. 37, 418 (1973).

[3] B. Welber, M. Cardona, C.K. Kim, S. Rodriguez, Phys. Rev. B 12, 5729 (1975). 